\title{
Vernizes intercalados: usos e virtudes
}

\section{Edson Motta Jr.}

Resumo: Este artigo descreve a aplicação de vernizes em sistemas intercalados no restauro de pinturas em vários momentos da história e em diversos países, e discute suas vantagens e desvantagens. Ele sugere também que a maior utilidade deste procedimento consiste em possibilitar uma cobertura e um brilho uniformes, e que estabilidade química e boas propriedades óticas podem ser obtidas também, associando-se Paraloid B72 a Regalrez 1094.

Palavras chave:Verniz; Sistemas de envernizado; Resinas; Saturação cromática; Intercalado.

\section{Barnices intercalados: usos y virtudes}

Resumen: Esto artículo describe sistemas de aplicación de barnices en los que se alternan resinas con propiedades diversas. Esos sistemas se han usado en distintos momentos de la historia y en diferentes países, y en esta publicacion se analizan sus ventajas y desventajas. El trabajo incide también en la gran virtud de esos métodos que es permitir una cobertura y brillo más uniformes, a la vez que se puede lograr estabilidad química y buenas propiedades ópticas si se hace la asociación de Paraloid B72 con Regalrez 1094.

Palabras clave: Barnices; Sistemas de barnizado; Resinas; Saturación cromática; Intercalado.

\section{Interlayered varnishes: uses and virtues}

Abstract: This paper describes and discusses the advantages and disadvantages of interlayered varnishing systems, used by paintings conservators in different moments in history and in countries with diverse conservation traditions. It also suggests that uniform coating and gloss are the main virtues of these systems, and that chemical stability and satisfying optical results can also be obtained by associating Paraloid B72 with Regalrez1094.

Keywords: Varnish; Varnishing systems; Resins; Colour saturation; Interlayer.

\section{Introdução}

Uma análise da literatura técnica sobre a prática de envernizado de pinturas dos últimos quarenta anos evidencia esforços da parte da comunidade profissional de cientistas e conservadores, no sentido de resolver as diversas imperfeições dos vernizes usados no restauro de pinturas.

Esses esforços se traduziram em pesquisas para a obtenção de resinas estáveis e oticamente apropriadas, as quais investigaram: a) a adequação de resinas desenvolvidas para fins industriais; $b$ ) a estabilização de vernizes já utilizados no restauro; c) e por fim, o desenvolvimento de sistemas de aplicação em que são intercaladas resinas com diferentes solubilidades para permitir a obtenção de superfícies homogeneamente recobertas, já que a absorção diferenciada dos vernizes pode deixar 
segmentos foscos que passam a contrastar com as áreas brilhantes. Esta última característica é frequentemente designada pelo termo "afundamento", uma tradução livre para o português do termo em inglês "sinking in". Este fenômeno ocorre mais frequentemente em pinturas que estão sendo submetidas ao restauro, as quais apresentam tintas lixiviadas, áreas de nivelamento com massas diversas e reintegrações com tintas com propriedades de impermeabilização variadas.

É importante ressaltar que a absorção diferenciada de um verniz pode ser desejável em algumas pinturas; portanto, as características uniformizadoras dos sistemas de envernizado são úteis apenas para obras nas quais se quer igualar o brilho da superfície.

Os sistemas de envernizado também são propostos por outras razões, dentre as quais, aumentar a estabilidade de uma resina natural, possibilitar a aplicação do verniz final por meio de pincel, e melhorar as propriedades óticas da pintura. Vernizes aplicados por aspersão tendem a ser mais homogêneos e menos contínuos, já que chegam à superfície da tinta em pequenas gotículas com pouco solvente. Quando é usado um solvente de evaporação rápida (ou quando a pistola tiver sido regulada para produzir um jato fino), a resina tende a chegar à tinta, semi-seca, e a formar uma superfície microscopicamente granulada, que reflete a luz incidente de maneira mais difusa e, assim, diminui a saturação das cores.

O presente artigo tem como tema a descrição desses métodos, já que suas versões modernas são úteis para a obtenção de bons resultados práticos, e ajudam a explicar a aparência característica das pinturas restauradas no contexto das diferentes tradições nacionais. Além disso, tratam-se de procedimentos relativamente desconhecidos da comunidade profissional, mesmo possuindo uma longa e importante história.

\section{Os sistemas de envernizado}

Na segunda metade do século XIX, houve um grande aumento na popularidade da resina Copal, usada como verniz e como aditivo para tintas a óleo (Swicklik 1993: 161). Nesse contexto surgiu um método de envernizado que envolvia a aplicação dessa resina diretamente sobre a tinta e, sobre ela, uma camada de verniz Mastic. [tabela 1] Acreditava-se que essa última, ao se deteriorar, poderia ser removida com relativa facilidade, e que a resina Copal, que se torna insolúvel rapidamente, protegeria as veladuras e outras sutilezas da pintura, que tão frequentemente sofrem danos durante as limpezas (Carlyle 2001: 57-65; Swicklik 1993: 160; Phenix 1993: 18). A lógica que fundamenta essa técnica seria impecável, se não fosse pelo intenso amarelecimento das resinas Copal, o que gerava a necessidade de futuras intervenções e, assim, submetia as obras aos riscos inerentes a esses procedimentos. Mérimée, nas suas próprias palavras, dá-nos uma indicação de como se deveria proceder: "A melhor maneira de preservar pinturas seria primeiramente envernizálas levemente com Copal e, quando essa camada estiver perfeitamente seca, aplicar sobre ela uma camada de resina Mastic. Em poucos anos essa se tornará amarelecida e esbranquiçada e poderá ser removida. O verniz Copal, sendo extremamente duro, não sofrerá pela remoção dessa cobertura, mas preservará a pintura tão bem que até as veladuras não poderão ser ameaçadas pela limpeza" (Mérimée 1839: 287). Não temos referências do uso deste método de envernizado em conservação, mas sua inclusão neste texto se justifica pelo fato de que ele foi idealizado com o objetivo de proteger a pintura de restauros futuros. 
Tabela 1: Sistema Mérimée

\begin{tabular}{|c|c|c|c|c|c|c|}
\hline \multicolumn{2}{|c|}{ SISTEMA MÉRIMÉE } & Resina & Concentração & Solvente & Aditivos & Aplicação \\
\hline \multirow{2}{*}{ Segunda camada } & Mastic & $20 \%$ & Terebintina & - & Com pincel \\
\cline { 2 - 6 } & Primeira camada & $\begin{array}{c}\text { Copal mais óleo } \\
\text { secante }\end{array}$ & - & Terebintina & - & Com pincel \\
\hline
\end{tabular}

Em 1940 no Manual on the Conservation of Paintings foram publicadas instruções para o uso de um sistema de envernizado específico para o restauro de pinturas, que envolvia a aplicação de emulsão de cera sobre um primeiro verniz de resina natural, para protegê-la da umidade, reduzir seu brilho e servir como camada de sacrifício, já que a cera pode ser removida facilmente quando suja, e reaplicada, a seguir, se necessário. (Ruhemann 1968: 273; Bradley 1950: 3.2; Abercauph 1996: 201205). [tabela 2] Nesse manual encontramos o seguinte parágrafo: "Esta aplicação final de uma camada de cera também parece ser o método mais recomendável a se adotar para o tratamento de qualquer tipo de verniz brilhante [...] [Ela] atribui à pintura um agradável acabamento semifosco e pode ser polida para formar uma camada uniforme e com um brilho completamente transparente por meio de um [polimento] com lã de algodão."

Ruhemann reconhece os méritos deste método, e o fato de que o mesmo não havia sido superado por ocasião da publicação de seu livro "The Cleaning of Paintings", em 1968. Por outro lado, o autor afirma que o uso da cera estava longe do ideal, já que ela capta poeira com facilidade e, consequentemente, reduz a transparência do verniz subjacente, especialmente quando este se encontra sobre tintas escuras, com craquelês e irregularidades acentuadas.

Tabela 2: Sistema Resina / Cera

\begin{tabular}{|c|c|c|c|c|c|c|}
\hline \multicolumn{2}{|c|}{$\begin{array}{c}\text { SISTEMA } \\
\text { RESINA / CERA }\end{array}$} & Resina & Soncentração & Aditivos & Aplicação \\
\hline $\begin{array}{c}\text { Segunda } \\
\text { camada }\end{array}$ & $\begin{array}{c}\text { Emulsão ou pasta de cera } \\
\text { de abelhas }\end{array}$ & - & $\begin{array}{c}\text { Terebintina ou } \\
\text { água }\end{array}$ & Com panos \\
\hline $\begin{array}{c}\text { Primeira } \\
\text { camada }\end{array}$ & $\begin{array}{c}\text { Resina (Mastic, Damar, } \\
\text { Cetônica) }\end{array}$ & $20 \%$ & Terebintina & Com pincel \\
\hline
\end{tabular}


Mais recentemente, no final dos anos 1970, um método mais sofisticado, que combinava duas resinas com propriedades diferentes, foi desenvolvido pelos restauradores e cientistas do Institut Royal du Patrimoine Artistique da Bélgica. A técnica consistia em uma primeira aplicação de resina Damar diretamente sobre a tinta e, a seguir, sobre ela, uma fina aspersão de Paraloid B-72 (etilmetacrilato/metilacrilato). [tabela 3] A função da resina natural era dar boa saturação às cores, e a função da resina acrílica era proteger o Damar da luz e da abrasão mecânica. A lógica desse procedimento foi primeiramente teorizada por Eddy De Witte e Miriam Goessens-Landrie (1978: 79) em um estudo no qual se investigou a influência da luz na aparência e na estabilidade dos vernizes.

Nicole Goetghbeur, restauradora de pinturas do IRPA, descreve a maneira pela qual esse sistema seria usado para o envernizado do "Levantamento da Cruz", de Peter Paul Rubens, exposto na Catedral de Antuérpia, e que estava sendo restaurado no ano de 1990: “O envernizado será feito em duas etapas, primeiro uma camada de verniz Damar, para unificar a totalidade da aparência e dar o máximo de profundidade à pintura, e depois uma fina camada protetora de Paraloid B-72". (Goetghebeur 1990: 1-5).

Em 1990, Bourdeau (1990: 45-6) publicou pesquisas usando a mesma sobreposição de resinas, adicionando, porém, ao Paraloid B72, aditivos bloqueadores de radiação ultravioleta. $O$ estudo concluiu que essa associação diminuía a velocidade da deterioração, mas não a impedia.

Tabela 3: Sistema IRPA

\begin{tabular}{|c|c|c|c|c|c|c|}
\hline \multicolumn{2}{|c|}{ SISTEMA IRPA } & Resina & Concentração & Solvente & Aditivos & Aplicação \\
\hline \multirow{2}{*}{$\begin{array}{c}\text { Segunda } \\
\text { camada }\end{array}$} & Paraloid B-72 & $5 \%$ & Xilenos & Por aspersão \\
\hline $\begin{array}{c}\text { Primeira } \\
\text { camada }\end{array}$ & Resina Damar & $10 \%$ & White spirits & Com pincel \\
\hline
\end{tabular}

Em Londres, os restauradores do Courtauld Institute of Art, desenvolveram também na década de 1970, um sistema que usava uma camada de Paraloid B72 sobre a tinta, seguida, após sua secagem, da aplicação por meio de pincel, de uma camada da resina Laropal K80. [tabela 4] Essa técnica era possível porque o verniz cetônico, diluído em hidrocarbonetos alifáticos, não dissolvia o Paraloid B72, solúvel somente em aromáticos. A razão para a sobreposição das duas resinas baseava-se na premissa de que, ficando o Paraloid B72, um polímero estável, diretamente em contato com a tinta, haveria sempre a garantia de fácil remoção do Laropal K80, mesmo se ele viesse a se tornar insolúvel no futuro; ${ }^{1}$ além disso, a aplicação de ambas as resinas por meio de pincel garantia um bom nivelamento final da superfície, e evitava, assim, a aparência microáspera, tão frequente nos vernizes aspergidos.

Seguindo essa lógica, era recomendado também que as reintegrações cromáticas fossem realizadas com Paraloid B72, para permitir o uso do Laropal K-80 sem o risco de remoção acidental 
das mesmas. Eventualmente, Regalrez 1094 e/ou a resina MS2A, materiais mais estáveis e também solúveis em hidrocarbonetos alifáticos, substituíram a resina cetônica nesse sistema.

Tabela 4: Sistema Courtauld

\begin{tabular}{|c|c|c|c|c|c|c|}
\hline \multicolumn{2}{|c|}{ SISTEMA COURTAULD } & Resina & Concentração & Solvente & Aditivos & Aplicação \\
\hline \multirow{2}{*}{ Segunda camada } & Laropal K80 & $20 \%$ & White spirits & Cera de abelha & Com pincel \\
\hline & Primeira camada & Paraloid B-72 & $10 \%$ & Xilenos & - & Com pincel \\
\hline
\end{tabular}

Simultaneamente, o Departamento de Conservação e Restauração do National Maritime Museum, em Greenwich, passou a usar um método de envernizado que combinava uma primeira aplicação de Laropal K80 acrescida de 10\% de cera microcristalina, diretamente sobre a tinta, seguido de uma segunda película - aspergida - do verniz cetônico Griffin, da Winsor \& Newton. [tabela 5] Esse procedimento baseava-se na crença de que a cera adicionada à resina que ficava diretamente sobre a tinta facilitaria a remoção futura do sistema, permitindo a permeação do solvente por sua estrutura, e que o verniz Griffin, por conter um percentual de óleo, atribuiria melhores propriedades óticas à pintura. Essa forma de envernizar dava à superfície uma aparência surpreendentemente sedosa, além de ser muito eficaz na função de saturar as áreas escuras. Podese teorizar que a presença do óleo como aditivo ao verniz Griffin retardava o tempo de secagem deste, possibilitando a formação de uma superfície mais nivelada e menos propensa à dispersão da luz incidente, aumentando assim os níveis de saturação cromática.

Tabela 5: Sistema Greenwich

\begin{tabular}{|c|c|c|c|c|c|c|}
\hline \multicolumn{2}{|c|}{$\begin{array}{c}\text { SISTEMA } \\
\text { GREENWICH }\end{array}$} & Resina & Concen-tração & Solvente & Aditivos & Aplicação \\
\hline \multirow{2}{*}{$\begin{array}{c}\text { Segunda } \\
\text { camada }\end{array}$} & $\begin{array}{c}\text { Griffin Picture } \\
\text { Varnish }\end{array}$ & $22 \%$ & White spirits & Óleo Stand & Por aspersão \\
\hline $\begin{array}{c}\text { Primeira } \\
\text { camada }\end{array}$ & Laropal K80 & $20 \%$ & White spirits & Cera de abelha & Com pincel \\
\hline
\end{tabular}


Do outro lado do atlântico, Mario Modestini, restaurador italiano radicado nos Estados Unidos, desenvolveu, na década de 1960, um sistema intercalado de envernizado que se difundiu entre os profissionais de Nova lorque, conquistando adeptos como Gustav Berger. Ele se baseava na combinação da capacidade de saturação da resina cetônica com as propriedades isolantes das resinas de acetato de polivinila. O procedimento envolvia a aplicação de uma primeira camada do Artists Retouching Varnish (Talens) ${ }^{2}$ e, quando esse estivesse completamente seco, uma aspersão de uma fina película de resina de PVA/ AYAB (e mais tarde Mowilith 20) com o propósito de formar uma camada isolante. As resinas de acetato de polivinila se dissolvem em solventes aromáticos ou para uso como verniz aspergido - em álcool etilico, e o verniz Artist Retouching Varnish é fornecido em solventes alifáticos. A seguir, voltava-se a aplicar do verniz da casa Talens que, devido à impermeabilidade do PVA, criava uma película contínua e uniforme sobre a pintura, reduzindo as áreas de absorção desigual (Dwayer 1992: 121-122). A sequencia poderia ser repetida conforme as exigências do restauro em curso, [Tabela 6] e a única ressalva que se faz a essa técnica está relacionada à notória tendência à insolubilidade da resina cetônica, que neste sistema fica diretamente em contato com a superfície da tinta.

A avaliação relativa ao amarelecimento desses vernizes foi feita por Dianne Dwyer ao revisitar pinturas restauradas por Mario Modestini, no final dos anos oitenta, para museus americanos. Ela menciona que: "Em uma pesquisa de pinturas em dezoito galerias regionais da Kress (Foundation), que foi levada a cabo há poucos anos, obras envernizadas usando esta técnica (PVA entre camadas de Talens) conservavam-se excepcionalmente bem. Depois de trinta anos, as superfícies mantinham um lustro agradável e o verniz não descoloriu perceptivelmente [...] em comparação, pinturas envernizadas poucos anos antes, pelos mesmos restauradores, usando Damar, mostraram amarelecimento significativamente maior" (Dwayer 1992: 121-2).

Tabela 6: Sistema Modestini

\begin{tabular}{|c|c|c|c|c|c|}
\hline $\begin{array}{l}\text { SISTEMA } \\
\text { MODESTINI }\end{array}$ & Resina & Concentração & Solvente & Aditivos & Aplicação \\
\hline $\begin{array}{l}\text { Terceira } \\
\text { camada }\end{array}$ & $\begin{array}{c}\text { Retouching Varnish } \\
\text { (Talens) }\end{array}$ & $25 \%$ & White spirits & Óleo de rícino & Com pincel \\
\hline $\begin{array}{l}\text { Segunda } \\
\text { camada }\end{array}$ & PVA / AYAB & $10 \%$ & $\begin{array}{l}\text { Xilenos } \\
\text { ou etanol }\end{array}$ & - & Por aspersão \\
\hline $\begin{array}{l}\text { Primeira } \\
\text { camada }\end{array}$ & $\begin{array}{l}\text { Retouching Varnish } \\
\text { (Talens) }\end{array}$ & $25 \%$ & White spirits & Óleo de rícino & Com pincel \\
\hline
\end{tabular}

Outro processo intercalado de envernizado, desenvolvido nos EUA, foi primeiramente apresentado por Eric Hulmer (1972: 145-7) no Congresso do International Institute for Conservation, realizado 
em Lisboa em 1972, e consiste na aplicação de Paraloid B72 sobre a pintura e, após a secagem deste, a aplicação de Paraloid B-67 (metacrilato de isobutila) como camada final. [tabela 7] A diferença de solubilidade entre essas duas resinas é novamente o princípio que rege o sistema já que o Paraloid $\mathrm{B} 67$ se dissolve em hidrocarbonetos alifáticos acrescidos de um pequeno percentual de aromáticos e, portanto, não tem a polaridade necessária para remover o Paraloid B72, que somente se dissolve em aromáticos puros. O mesmo princípio se aplica, caso a reintegração seja realizada com tintas aglutinadas com Paraloid B72 ou PVA: o Paraloid B67 pode ser usado como verniz final, aplicado por pincel, sem dissolver a reintegração. Além disso, conforme observado acima, o uso do Paraloid B72 garantia a reversibilidade futura do verniz, já que esse ficava em contato direto com a tinta. Como nos outros métodos semelhantes a esse, o número de camadas intercaladas das duas resinas variava conforme as exigências de cada pintura em restauro.

Tabela 7: Sistema Hulmer

\begin{tabular}{|c|c|c|c|c|c|c|}
\hline \multicolumn{2}{|c|}{ SISTEMA HULMER } & Resina & Concentração & Solvente & Aditivos & Aplicação \\
\hline \multirow{2}{*}{$\begin{array}{c}\text { Segunda } \\
\text { camada }\end{array}$} & $\begin{array}{c}\text { Paraloid } \\
\text { B-67 }\end{array}$ & $10 \%$ & White spirits & - & Com pincel \\
\hline $\begin{array}{c}\text { Primeira } \\
\text { camada }\end{array}$ & $\begin{array}{c}\text { Paraloid } \\
\text { B-72 }\end{array}$ & $10 \%$ & Xilenos & Com pincel \\
\hline
\end{tabular}

Mais recentemente, a introdução da resina hidrocarbônica Regalrez 1094 como verniz para conservação e restauração, deu a oportunidade de se intercalar pela primeira vez, materiais estáveis em uma mesma técnica de envernizado. Essa resina foi pesquisada por René de la Rie e Christopher Mc Glinckey (1989: 53-70; 1990: 563-7; 1993: 566-73) que buscavam um produto que reunisse estabilidade e propriedades óticas similares às das resinas naturais; porém, a baixa viscosidade de Regalrez, assim como a grande atração intermolecular entre a resina e os solventes nos quais é diluído, faz com que ele demore a atingir o ponto de no flow e, portanto, sua penetração na estrutura da tinta é muito maior do que a que se observa com resinas similares.

A noção de no flow refere-se ao momento em que um verniz em solução tem sua viscosidade aumentada na mesma proporção da evaporação do solvente e, em consequência disso, sua penetração pela estrutura irregular e capilar da tinta diminui, e eventualmente cessa, deixando de preencher todos os microespaços mais profundos na estrutura da tinta. [ilustracao 1] Os vernizes de baixa viscosidade, os diluídos em solventes de evaporação lenta, ou aqueles com os quais a resina em solução e o solvente têm grande afinidade química, como as resinas hidrocarbonicas, demoram mais a atingir o ponto de no flow. Este tema foi discutido sob outra ótica por Rene de la Rie $(1987,1-13)$ 


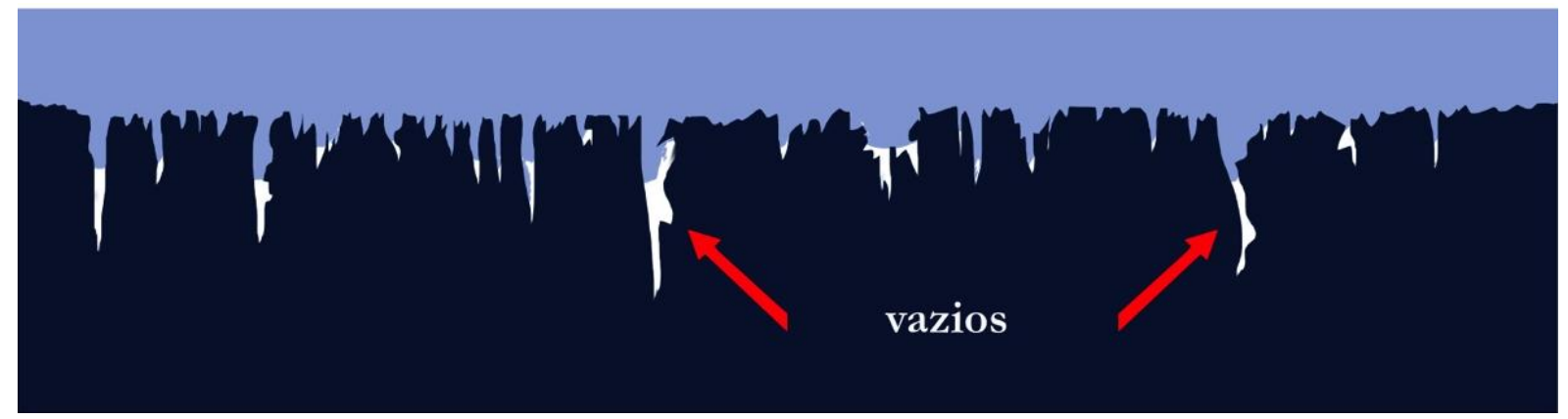

Ilustração 1: Modelo ilustrativo da hipótese de no flow

Em decorrência disso, não se forma uma película continua sobre as áreas de tinta mais porosas (com menor percentual de aglutinante ou formada com pigmentos mais granulados). Por outro lado, nas áreas com maior percentual de aglutinante e/ou com pigmentos menores e mais homogêneos, o verniz produz um estrato definido e homogêneo na superfície da tinta; portanto, mais brilhante. Como consequência, as superfícies envernizadas ficam, com frequência, irregulares, o que produz um desconforto visual incompatível com a adequada apreciação de uma pintura.

Este obstáculo, porém, pode ser superado aplicando-se o Regalrez 1094 diretamente sobre a tinta, por meio de pincel. Esta forma de aplicação garante boa saturação das cores, especialmente quando se trata de pinturas muito lixiviadas por solventes em limpezas anteriores e/ou com resíduos de vernizes antigos. Após sua secagem, Paraloid B72 é aspergido para formar uma película isolante, preparatória para uma segunda aplicação de Regalrez, que irá atribuir bom leveling final ao sistema, e deixar sobre a superfície uma resina de alta temperatura de transição vítrea. O termo leveling é usado para descrever a propriedade que possuem os vernizes de baixa viscosidade, de formarem entre um pico da textura e outro, uma superfície mais lisa que reflete menos luz difusa. A representação gráfica do leveling pode ser vista abaixo. [ilustração 2]

A adição de até $4 \%$ da borracha polimérica sintética Kraton 1657, também pode ser incorporada a esta última camada para dar-lhe uma aparência menos brilhante e mais suave [tabela 8] e, além disto, a sequencia de resinas pode ser repetida até a obtenção de resultados óticos e estéticos desejados ${ }^{3}$.

Tabela 8: Sistema Regalrez

\begin{tabular}{|c|c|c|c|c|c|c|}
\hline \multicolumn{2}{|c|}{$\begin{array}{l}\text { SISTEMA } \\
\text { REGALREZ }\end{array}$} & Resina & Concentração & Solvente & Aditivos & Aplicação \\
\hline & $\begin{array}{l}\text { Terceira } \\
\text { camada }\end{array}$ & Regalrez 1094 & $25 \%$ & White spirits & $\begin{array}{c}\text { Tinuvin } 292 \text { e } \\
\text { Kraton } 1657\end{array}$ & Por aspersão \\
\hline & $\begin{array}{l}\text { Segunda } \\
\text { camada }\end{array}$ & $\begin{array}{c}\text { Paraloid } \\
\text { B-72 }\end{array}$ & $5 \%$ & Xilenos & - & Por aspersão \\
\hline & $\begin{array}{l}\text { Primeira } \\
\text { camada }\end{array}$ & Regalrez 1094 & $25 \%$ & White spirits & Tinuvin 292 & Com pincel \\
\hline
\end{tabular}


01
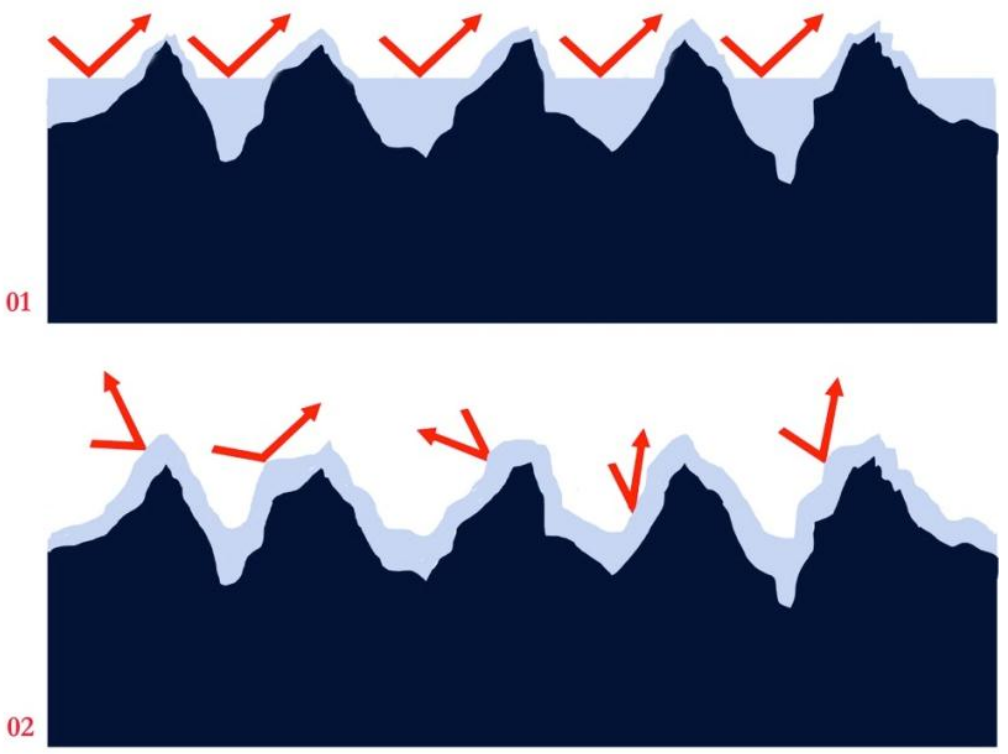

Ilustração 2: Modelo gráfico de leveling

01. Leveling de vernizes poliméricos

02. Conformação à topografia dos vernizes poliméricos

\section{Conclusão}

Os sistemas de aplicação de resinas intercaladas são recursos técnicos usados na história recente da conservação e do restauro, com o objetivo principal de melhorar as propriedades óticas e a aparência dos vernizes, além de facilitar sua remoção no futuro. Os diferentes métodos desenvolvidos a partir da publicação do Manual on the Conservation of Paintings em 1940, são evidências do esforço e da criatividade neste sentido; porém, a grande maioria deles apresentava alguma insuficiência técnica relacionada aos materiais disponíveis em cada momento histórico. Com a introdução do Regalrez 1094 na prática dos Conservadores-Restauradores, os problemas relacionados à estabilidade cromática e à reversibilidade foram superados; por outro lado, aumentaram as imperfeições relacionadas à desigualdade de brilho e à saturação, o que cria problemas estéticos que só podem ser ultrapassados, parcialmente, com a adição de polímeros específicos (Kraton 1657), aspersão da resina com solventes de evaporação rápida, ou ainda, de forma mais eficiente, com sistemas que intercalam Regalrez 1094 e Paraloid B724.

\section{Notas}

[1] Desde 1963, com as pesquisas de Gerry Thomson, sugeria-se que as resinas cetônicas eram de difícil remoção com o tempo. A confirmação dessa instabilidade veio somente em 1972 (Feller, 1972:7578). Estas resinas, infelizmente, formam ainda hoje, a base de muitos vernizes comerciais. As informações sobre esse sistema assim como o conhecimento do sistema Greenwich foram obtidas pelo autor quando este foi aluno do curso de formação em conservação e restauro do Courtauld Institute of Art, Londres, entre 1982/84. Estes sistemas são de interesse histórico, já que muitos caíram em desuso. 
[2] No início dos anos 1990, Gustav Berger substituiu essa resina cetônica por uma resina hidrocarbônica estável: Escorez 5380 ( Swicklik; Berger; Berger 1998: 125-129).

[3] Esta sequencia produz excelentes resultados, também usando-se a resina cetônica hidrogenada MS2A.

[4] Este sistema de envernizado tem sido usado com regularidade no Brasil há vinte anos, e até a presente data, as pinturas que puderam ser examinadas encontram-se em bom estado.

\section{Bibliografia}

ABERCAUPH, C. (1996) Painting Conservation Catalog, Varnishes and Surface Coatings (volume 1). Washington: AIC.

BOURDEAU, J. (1990). "A Further examination of the barrier properties of Tinuvin 327 ultraviolet absorber in the of dammar films". Em: Cleaning retouching and coatings: Technology and practice for easel paintings and polychrome sculpture. Preprints of the contributions to the Brussels Congress. London: IIC.

BRADLEY, M. (1950). The Treatment of Pictures. Cambridge, USA: Cosmos Press, Inc.

CARLYLE, L. A. (2001).The Artist's Assistant, Oil painting Instruction Manuals and Handbooks in Britain 1800-1900. With Reference to Selected Eighteenth-century Sources. New York: Archetype Publications.

DE LA RIE, E. R. (1987). “The influence of varnishes on the appearance of paintings.” Studies in Conservation 32-1.

DE LA RIE, E. R. (1990). "New synthetic resins for picture varnishes." Em: Cleaning Retouching and Coatings: Technology and Practice for Easel Paintings and Polychrome Sculpture. Preprints of the contributions to the Brussels Congress, London: IIC.

DE LA RIE, E. R. (1993). "Polymer additives for synthetic low-molecular-weight varnishes". Em Committee for Conservation, 10th Triennial Meeting, Washington: ICOM.

DE WITTE, E. e GOESSENS-LANDRIE, M. (1978-79). "The influence of light on the appearance and the stability of varnishes". Bulletin du Institut Royal du Patrimoine Artistique. Brussels.

DWYER, D. (1992). "A Varnishing technique used by Mario Modestini". Em: Paintings Specialty Group Postprints, Twentieth Annual Meeting of the American Institute for Conservation of Historic and Artistic Works, AIC.

FELLER, R. L. (1972). "Problems in the Investigation of Picture Varnishes." Em Conservation of Paintings and the Graphic Arts. International Institute for Conservation, Lisbon Congress. London: Butterworths.

FELLER, R., STOLOW, N. e JONES, E. (1985). On Picture varnishes and their solvents. Washington, DC: National Gallery of Art.

GOETGHEBEUR, N. (1990). "Preliminary study and approach to the cleaning of "The raising of the cross" by Peter Paul Rubens in the Antwerp Cathedral." Em: Cleaning, retouching and coatings: Technology and Practice for Easel Paintings and Polychrome Sculpture. Preprints of the Contributions to the Brussels Congress. London: International Institute for Conservation of Historic and Artistic Works.

HULMER, E. C. (1972). "Notes on the formulation and application of acrylic coating." Em: Conservation of Paintings and the Graphic Arts, Lisbon Congress, International Institute for Conservation of Historic and Artistic Works, 1972. London: Butterworths. 
MAYER, L. (1998) Painting Conservation Catalog, Varnishes and Surface Coatings (volume 1) USA:AIC.

MERIMEE, M.J.-F.-L. (1839). The Art of painting in oil, and in fresco: Being a history of the various processes and materials employed, from its Discovery, by Hubert and John Van Eyck, to the present time. London: Whittaker \&Co.

PHENIX, A. (1993). "Artists' and conservation varnishes: An historical overview". Em: Varnishing: Theory and practice. The Association of British Picture Restorers, Fiftieth Anniversary Conference.

RUHEMANN, H. (1968) The cleaning of paintings: problems and potentialities. London: Faber \& Faber.

SWICKLIK, M. (1993). “French painting and the use of varnish, 1750-1900.” Em: Conservation Research, Studies in the History of Art 41, Monograph Series II. Washington, D.C.: National Gallery of Art.

SWICKLIK, M; BERGER,G; BERGER,M. (1998) Paintings Conservation Catalog, Varnishes and Surface Coatings. Washington: AIC.

THOMSOM, G. (1963). “New Picture Varnishes.” Em Recent Advances in Conservation. London: Butterworths

WHITTEN, J. (1998) Painting Conservation Catalog, Varnishes and Surface Coatings. Washington: AIC

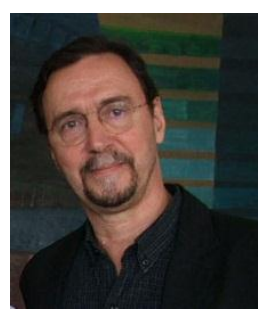

Edson Motta, Jr.

edsonmotta@terra.com.br

Edson Motta, Jr. é conservador-restaurador de pinturas e professor da Universidade Federal do Rio de Janeiro. Iniciou sua formação com Edson Motta (Snr.) em 1974, e de 1982 a 1985 estudou no Courtauld Institute of Art, Universidade de Londres. Em 1989/90 foi Conservador-Restaurador visitante no Departamento de Conservação de Pinturas do J. Paul Getty Museum em Malibu, USA, e concluiu o Doutorado na Universidade Politécnica de Valência, Espanha, em 2004. Devo a Maria Cristina da Silva Graça as opiniões criticas e revisão do texto. 